\title{
Quantum Magnetism in the Paratacamite Family: Towards an Ideal Kagomé Lattice
}

\author{
P. Mendels, ${ }^{1}$ F. Bert, ${ }^{1}$ M. A. de Vries, ${ }^{2}$ A. Olariu, ${ }^{1}$ A. Harrison, ${ }^{2}$ F. Duc,${ }^{3}$ J. C. Trombe,${ }^{3}$ \\ J. S. Lord, ${ }^{4}$ A. Amato, ${ }^{5}$ and C. Baines ${ }^{5}$ \\ ${ }^{1}$ Laboratoire de Physique des Solides, UMR CNRS 8502, Université Paris-Sud, 91405 Orsay, France \\ ${ }^{2}$ School of Chemistry and CSEC, The University of Edinburgh, Edinburgh EH9 3JZ, United Kingdom \\ ${ }^{3}$ Centre d'Élaboration des Matériaux et d'Études Structurales, CNRS UPR 8011, 31055 Toulouse, France \\ ${ }^{4} I S I S$ Facility, Rutherford Appleton Laboratory, Chilton, Didcot, Oxon OX11 OQX, United Kingdom \\ ${ }^{5}$ Laboratory for Muon Spin Spectroscopy, Paul Scherrer Institute, CH-5232 Villigen PSI, Switzerland
} (Received 19 October 2006; published 13 February 2007)

\begin{abstract}
We report muon spin rotation measurements on the $S=1 / 2\left(\mathrm{Cu}^{2+}\right)$ paratacamite $\mathrm{Zn}_{x} \mathrm{Cu}_{4-x}(\mathrm{OH})_{6} \mathrm{Cl}_{2}$ family. Despite a Weiss temperature of $\sim-300 \mathrm{~K}$, the $x=1$ compound is found to have no transition to a magnetic frozen state down to $50 \mathrm{mK}$ as theoretically expected for the kagomé Heisenberg antiferromagnet. We find that the limit between a dynamical and a partly frozen ground state occurs around $x=$ 0.5 . For $x=1$, we discuss the relevance to a singlet picture.
\end{abstract}

DOI: 10.1103/PhysRevLett.98.077204

The idea of destabilizing a Néel state in favor of a spin liquid state by means of geometrical frustration was proposed long ago by Anderson in the context of triangular $S=1 / 2$ antiferromagnets (AF) [1]. The underlying concept of a resonating valence bond (RVB) state, built from a macroscopic number of singlets, has proven since then to be a very rich theoretical playground and has indeed been advocated in the important context of hole-doped quantum AF such as High- $T_{c}$ cuprates, spin ladders, etc. The simpler and fundamental case of geometrically frustrated AF insulators has been deeply revisited for the last 15 years. On a theoretical ground, there is now a consensus that a RVB state should be the ground state for $S=1 / 2$ kagomé $[2,3]$ or pyrochlore [4] Heisenberg AF (HAF), but not for the more connected triangular lattice. The most striking results from exact diagonalizations of the $S=1 / 2$ kagomé HAF are the fairly small gap value $<J / 20$ and the existence of a continuum of low lying singlet excitations, between the ground state and the first excited triplet [2,5].

Nonconventional dynamics were experimentally revealed in various corner-sharing AF [6-8], which yielded the best support to a resonatinglike ground state but no satisfactory experimental realization has been available yet to check in detail the $T=0$ theoretical predictions for $S=$ $1 / 2$ systems, e.g., the existence of a singlet-triplet gap. Indeed, in a context where all perturbations such as single ion, exchange anisotropy, or spinless defects are relevant to induce various marginal orderings, no material has fulfilled the condition of a $S=1 / 2$ perfect kagomé HAF lattice. In $\left[\mathrm{Cu}_{3}(\mathrm{titmb})_{2}\left(\mathrm{OCOCH}_{3}\right)_{6}\right] \mathrm{H}_{2} \mathrm{O}$ second and third neighbor interactions play a dominant role [9,10]. Volborthite is maybe the first $S=1 / 2$ kagomé lattice [11], although one suspects spatially anisotropic near neighbor interactions; it undergoes a transition to a peculiar spin glass state around $1 \mathrm{~K}$ and has persisting $T \rightarrow 0$ dynamics $[12,13]$. As for $3 \mathrm{D}$ structures, rare earth pyrochlores are up to now recognized as perfect corner-sharing lattices, but most of their fascinating properties are governed by the delicate
PACS numbers: 75.30.-m, 75.40.Cx, 76.75.+i

balance between magnetocrystalline anisotropy, exchange, and, due to a large $S$ value, dipolar interactions. To our knowledge, clinoatacamite $\mathrm{Cu}_{4}(\mathrm{OH})_{6} \mathrm{Cl}_{2}$ would be the first example approaching a $S=1 / 2\left(\mathrm{Cu}^{2+}\right)$ Heisenberg pyrochlore lattice, but it has a distorted lattice and possibly a ferromagnetic coupling along the $c$ axis. A recent muon spin rotation $(\mu \mathrm{SR})$ study clearly revealed two magnetic transitions, one around $18 \mathrm{~K}$ and the other one around $6 \mathrm{~K}$, to a state where a weak ferromagnetic moment appears and where dynamics persist down to $T=0$ [14].

Quite recently, the $x=1$ compound of the $\mathrm{Zn}$ paratacamite family $\mathrm{Zn}_{x} \mathrm{Cu}_{4-x}(\mathrm{OH})_{6} \mathrm{Cl}_{2}$ has been revealed as a "structurally perfect $S=1 / 2$ kagomé antiferromagnet" [15]. It can be viewed as a double variant of the parent compound clinoatacamite $(x=0)$ : First, the symmetry relaxes from monoclinic to rhombohedral $(R \overline{3} \mathrm{~m})$ around $x=0.33$, leading to a perfect kagomé lattice in the $a-b$ plane. Then, in the $c$-elongated $x>0.33$ pyrochlore structure, the magnetic bridge along the $c$ axis between $a-b$ kagomé planes is progressively suppressed by replacing the apical $\mathrm{Cu}^{2+}$ by a nonmagnetic $\mathrm{Zn}^{2+}$. At the composition $x=1$, the $S=1 / 2 \mathrm{Cu}^{2+}$ ions lie at the vertices of a perfect kagomé lattice. Whereas macroscopic susceptibility indicates that the $\mathrm{Cu}-\mathrm{Cu}$ antiferromagnetic interactions yield a Weiss temperature $\theta_{\mathrm{CW}} \sim-300 \mathrm{~K}$, no signature of a transition was found down to $1.8 \mathrm{~K}$.

In this Letter, we present the first sub-Kelvin magnetic study of $\mathrm{ZnCu}_{3}(\mathrm{OH})_{6} \mathrm{Cl}_{2}$ using $\mu \mathrm{SR}$, which is very sensitive to any freezing and perfectly suited to track persisting $T=0$ dynamics. We find neither evidence of a magnetic transition nor a drastic slowing down of the dynamics down to $50 \mathrm{mK}$, which differs markedly from the other existing geometrically frustrated magnets. We also track the crossover from a paramagneticlike ground state to a $T=0$ magnetic frozen state from $x=1$ to $x=0$.

Pure samples of $0.15 \leq x \leq 1.00$ were prepared using the hydrothermal method as described in [15]. To make the deuterated analog of the $x=1$ phase (D sample) the entire 
reaction was carried out in a nitrogen filled glove bag, deuterated Copper Carbonate was prepared, and 99\% $\mathrm{D}_{2} \mathrm{O}$ was used throughout. The purity of the samples and the $\mathrm{Cu}$-to- $\mathrm{Zn}$ ratio were verified $(\approx \pm 1 \%$ ) with powder $\mathrm{x}$ ray diffraction and inductively coupled plasma-atomic emission spectroscopy. SQUID tests were performed and gave results in perfect agreement with those of Ref. [15]. For $x=1$, we find $\theta_{\mathrm{CW}}=-300(20) \mathrm{K}$ and we detect a small Curie term which would correspond to a maximum of $6 \% S=1 / 2$ free spins out of the total $\mathrm{Cu}$ amount. In all our $x \leq 1$ samples a ferromagnetic frozen component appeared whose weight was found negligible for $x=1$ as in [15], and increased drastically for $x<0.5$.

$\mu \mathrm{SR}$ measurements were performed on $x=0.15-1.0$ $\mathrm{Zn}$ contents, in zero field (ZF), longitudinal applied field (LF) or weak transverse field (WTF), with respect to the muon initial polarization. Because of their positive charge, implanted muons come at rest in well-defined sites where their electrostatic energy is minimized, i.e., nearby $\mathrm{Cl}^{-}$and $\mathrm{OH}^{-}$. In a paramagnetic state, electronic moments fluctuate fast $\left(\sim 10^{-12} \mathrm{~s}\right)$ on the $\mu$ SR time scale and the field sensed by the muons has only a nuclear origin with a typical value of a few $\mathrm{G}$.

We first focus on the low- $T$ state for the "perfect kagomé" composition, $x=1$ ( $T=50 \mathrm{mK}$, Fig. 1). Damped ZF oscillations, corresponding to a few $\mathrm{G}$ field are evident for the $\mathrm{H}$ sample and were observed at all $T$, but disappear for the D sample. They are therefore clearly associated with the $\mathrm{H}$ nucleus. This is evidence for the formation of a $\mathrm{OH}-\mu$ complex where the muon binds to a hydroxyl group in the $\mathrm{H}$ sample [16]. The signature of such a complex lies in oscillations of the polarization. The characteristic frequency $\omega_{\mathrm{OH}}$ of the time evolution of the polarization $\mathrm{P}_{\mathrm{OH}}(t)$ can be calculated by modeling the dipolar interaction between the proton and the muon moments [17]. In the D sample, the frequency $\omega_{\mathrm{OD}}$ is too small (nuclear mo-

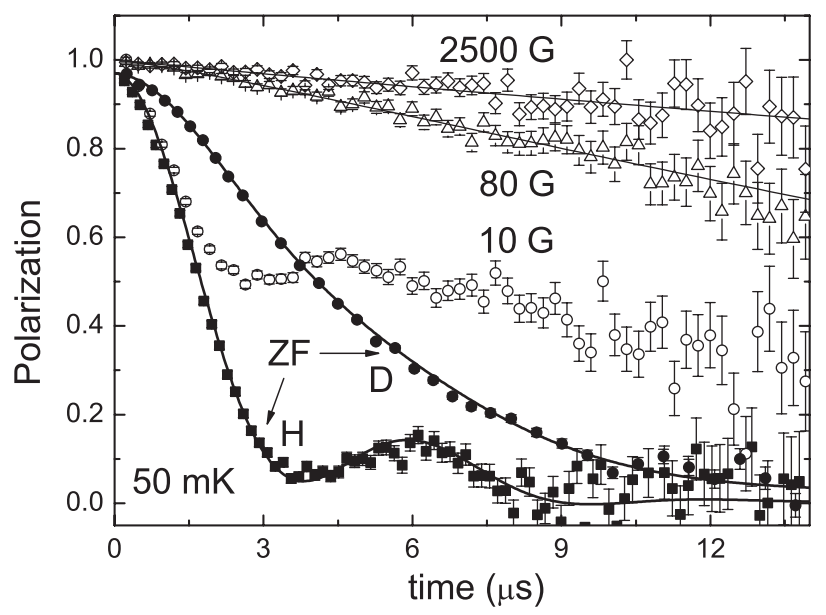

FIG. 1. $\mu^{+}$polarization in $x=1$ compound, either in zero field ( $\mathrm{H}$ and $\mathrm{D}$ samples) and under an applied field (H sample). No static internal field other than of nuclear origin is detected and a weak dynamical relaxation is observed. ments ratio $\mu_{D} / \mu_{H}=0.153$ ) and the oscillating pattern escapes our 0-16 $\mu$ s time window. As expected in a static case, a LF approximately a few tens of G, much larger than nuclear dipole fields, decouples nearly completely the relaxation, except a small relaxing tail which is found identical in 80 and $10 \mathrm{G}$ data. The latter can be safely attributed to a dynamical relaxation, due to fluctuations of the electronic moments, which only starts decoupling for much larger LF such as $2500 \mathrm{G}$. In addition, we do not find any change of the oscillations with $T$, which evidences that there is no static field other than the dipolar field from nuclei. We can qualitatively conclude that (i) no freezing and (ii) no drastic slowing down such as that observed in kagomé-like compounds $[6,8,12,13]$ occur even at $50 \mathrm{mK}$.

In order to refine this qualitative statement, the $\mathrm{ZF}$ polarization for the $\mathrm{H}$ sample was fitted to the sum of the two expected contributions,

$$
P_{z}(t)=\left[\mathrm{P}_{\mathrm{OH}}(t) e^{-\left(\gamma_{\mu} \Delta_{\mathrm{OH}} t\right)^{2} / 2}+\mathrm{KT}_{\mathrm{Cl}}(t)\right] e^{(-\lambda t)^{\alpha}} .
$$

The Gaussian damping $\Delta_{\mathrm{OH}}$ originates from nuclei surrounding the $\mathrm{OH}-\mu$ complex. $\mathrm{KT}_{\mathrm{Cl}}(t)$ is the well established Kubo-Toyabe function, Gaussian at early times used here for the $\mathrm{Cl}^{-}$site $(\sim 10 \%-20 \%)$ where the relaxation is due to surrounding $\mathrm{Cu}$ and $\mathrm{H}$ nuclei. In addition, a small dynamical relaxation was allowed through the stretched exponential overall multiplying factor [18]. All the static values used in the fits were fixed by a high statistics run at $1.5 \mathrm{~K}$ and found identical for higher $T$ values and other $x$ values - provided the samples are in a paramagnetic state. From the value of $\omega_{\mathrm{OH}}$, we find $H_{\mathrm{OH}}=7.6(2) \mathrm{G}$, and the field distributions are respectively $\Delta_{\mathrm{OH}}=2.2(3) \mathrm{G}$ and $\Delta_{\mathrm{Cl}}=1.7(5) \mathrm{G}$ for $\mathrm{OH}^{-}$and $\mathrm{Cl}^{-}$sites, typical of dipolar fields induced by nearby $\mathrm{H}, \mathrm{Cu}$, and $\mathrm{Cl}$ nuclei. The D-sample data can be consistently analyzed using $H_{\mathrm{OD}}=$ $H_{\mathrm{OH}} \times \frac{\mu_{D}}{\mu_{H}}$ and similar $\Delta$ values. At $50 \mathrm{mK}$ we found identical $H_{\mathrm{OH}}$ values and only the dynamical relaxation was found to slightly increase.

The absence of variation of the frequency of the oscillation with $T$ on the $\mathrm{H}$ sample demonstrates that although $\theta_{\mathrm{CW}} \sim-300 \mathrm{~K}$, the sample does not freeze into a magnetic state down to the lowest $T$ of $50 \mathrm{mK}$. Indeed, in the case of a magnetic freezing, the oscillation due to the $\mathrm{OH}-\mu$ complex would be swamped by the relaxation due to electronic moments. An upper value of the electronic field of $\sim 0.3 \mathrm{G}$ corresponds to the error bars of our fits. From the comparison with the $\sim 500 \mathrm{G}$ field found at the muon site in $\mathrm{Cu}_{4}(\mathrm{OH})_{6} \mathrm{Cl}_{2}$, one obtains an upper bound of $6 \times 10^{-4} \mu_{B}$ for the $\mathrm{Cu}^{2+}$ frozen moment.

We now turn to the discussion of the small dynamical effects observed on the long time tail of the muon polarization. We tracked its $T$ evolution by applying a $80 \mathrm{G}$ longitudinal field, large enough to decouple the static nuclear fields. In the left panel of Fig. 2, one can directly notice on the D-sample raw data a small increase of the muon relaxation between 1.5 and $50 \mathrm{mK}$. The values of $\lambda$ extracted from stretched exponential fits are plotted in the 
right panel of Fig. 2. The modest increase of $\lambda$ is found to occur around $1 \mathrm{~K}$. The stretched exponent $\alpha$ was also found to increase from 1 to 1.5 in this $T$ range which reminds one of the unconventional undecouplable Gaussian $(\alpha=2)$ observed in a broad variety of geometrically frustrated magnets (GFM) $[6,8]$.

In the simplest model of a single time relaxation process, $\lambda$ is related to the fluctuation frequency $\nu$ and the fluctuating electronic field $H_{\text {fluct }}$ by $\lambda=2 \gamma_{\mu}^{2} H_{\text {fluct }}^{2} \nu /\left(\nu^{2}+\gamma_{\mu}^{2} H_{\mathrm{LF}}^{2}\right)$. From fits of the 80 and $2500 \mathrm{G}$ data at $50 \mathrm{mK}$ (see Fig. 1), we get $\nu \sim 150 \mathrm{MHz}$ and $H_{\text {fluct }} \sim 18 \mathrm{G}$. This fluctuating field is much smaller than the static field detected in the frozen phase of the $x=0$ compound $(\sim 500 \mathrm{G})$ or the mere estimate of the dipolar field created by one $\mathrm{Cu}$ spin at the oxygen site $(\sim 2300 \mathrm{G})$. Since muons are only sensitive to slowly fluctuating spins which are not paired in a singlet state, we can safely conclude that most of the $\mathrm{Cu}$ spins appear to be inactive at $50 \mathrm{mK}$, i.e., either in a singlet state, or too fast fluctuating to be sensed by the muon. Slowly fluctuating defects are therefore responsible for the small relaxation we observe.

In order to make the bridge between the fully fluctuating ground state observed for $x=1$ and the freezing observed for $x=0$, we performed a detailed $T$ study of the evolution of the magnetic properties between these two compositions. In Fig. 3, we plot the typical polarizations obtained at $T \lesssim 1.6 \mathrm{~K}$, representative of the ground state. The evolution is best followed starting from $x=0$, where the freezing of electronic moments corresponds to well-defined spontaneous oscillations of the polarization associated with the precession of the $\mu^{+}$in internal fields $\sim 500 \mathrm{G}$ [19] (Fig. 3, inset). For $x=0.15$, the oscillations become less evident and a substantial slowly relaxing component appears. The weight of the latter keeps increasing up to $x=0.5$, at the expense of the fast front end (Fig. 3, main panel). The $x=0.66$ sample was found to have a dominant dynamical character down to $50 \mathrm{mK}$, which we probed through LF measurements such as described for $x=1$. For
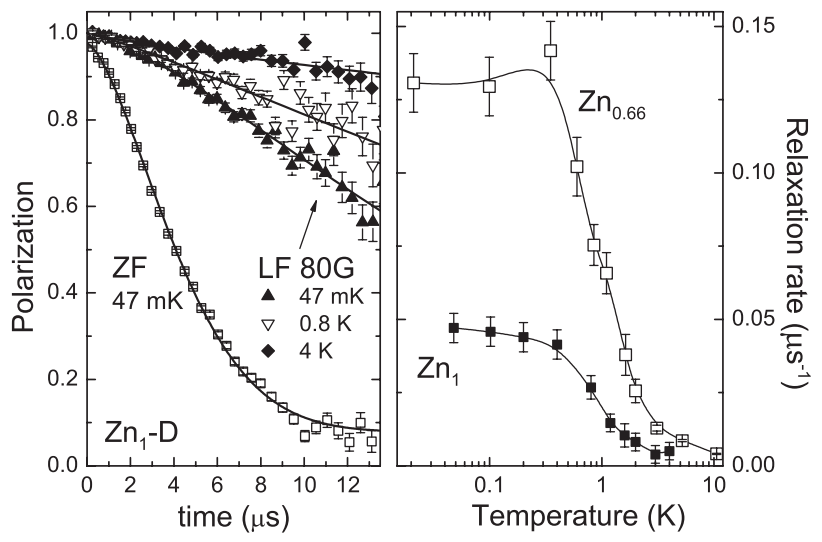

FIG. 2. Left panel: time evolution of the polarization for the $x=1 \mathrm{D}$ sample in $\mathrm{ZF}$ and LF. The slowing down observed below $1 \mathrm{~K}$ is more pronounced for $x=0.66$ than for $x=1$ (right panel). this composition, the slowly oscillating pattern of the $\mathrm{OH}-\mu$ complex is also partially recovered. The slowing down of the dynamics, more pronounced than for $x=1$, is clearly identified around $1 \mathrm{~K}$ (Fig. 2, right panel).

For $x \leq 0.5$, the ZF data were fitted to a superposition of a fast relaxing $(x \geq 0.33)$ or oscillating $(x \leq 0.15)$ component corresponding to the frozen fraction and a paramagnetic term given by Eq. (1), where all the static fields were kept at the values found at high $T$ consistent with those for $x=1$ (Fig. 3). The $T$ variation of the frozen fraction, determined through $20 \mathrm{G}$ WTF measurements, is plotted in Fig. 4. For $x=0.15$, two transitions clearly occur around 18 and $6 \mathrm{~K}$ but, at variance with the $x=0$ sample, correspond to different frozen fractions. For the $x=0.33$ and 0.5 sample, the $18 \mathrm{~K}$ transition disappears, which therefore rather seems typical of the monoclinic phase $(x \leq 0.33)$. A very broad transition is still observed around the same $T \sim 6 \mathrm{~K}$ and with very different frozen fractions. The change in the shape of the transition curves indicates that no macroscopic phase segregation occurs but rather that for $x \leq 0.5-0.6$, islands freezing around $6 \mathrm{~K}$ coexist with paramagnetic ones which have slower dynamics than for $x=1$. The low- $T$ fractions are represented in the inset versus $x$ and clearly indicate a change of behavior from partially static to dominantly dynamical behavior around $x=0.5-0.6$. This certainly relates to the $x=0.5$ percolation threshold between a limit of decoupled kagomé planes and a case where all $\mathrm{Cu}^{2+}$ on a triangle belong to at least one fully occupied (elongated) pyrochlore pattern. Above $x=0.5-0.6$, the dynamics is likely inhomogeneous due to random local variations in $\mathrm{Cu}$ occupancy of the $\mathrm{Zn}$ site. The extent of this region signals, in turn, that the physics of the kagomé planes is only little perturbed through $\mathrm{Cu}-\mathrm{Cu}$ interactions along the $c$ axis.

$\mathrm{ZnCu}_{3}(\mathrm{OH})_{6} \mathrm{Cl}_{2}(x=1)$ represents the first example of a kagomé system without any 3D freezing down to temperatures as low as $1.6 \times 10^{-4} \mathrm{~J}$. Its low- $T$ behavior differs widely from the cases of kagomé bilayers of the long

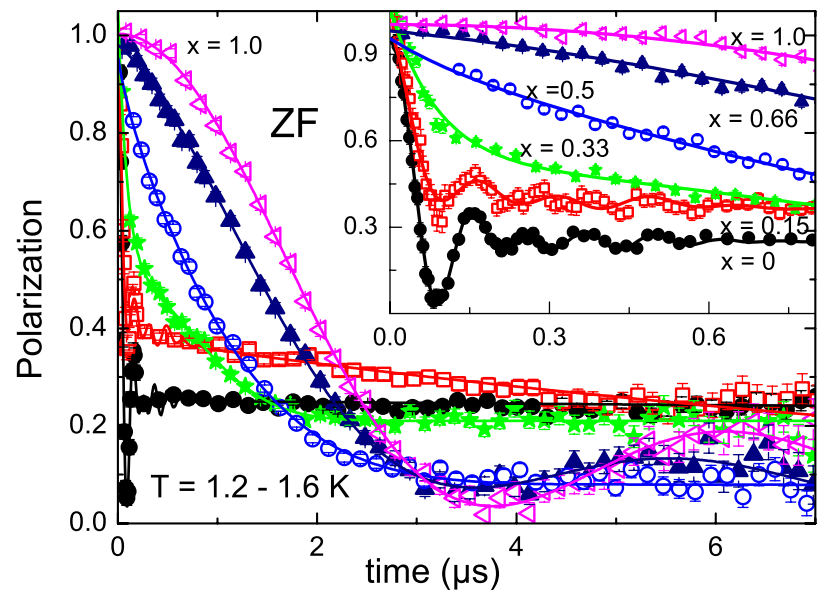

FIG. 3 (color online).$\quad \mu^{+}$polarization [23] plotted versus time for $x=0,0.15,0.33,0.5,0.66,1.0$. Lines are for fits described in the text. The data for $x=0$ are from [19]. 


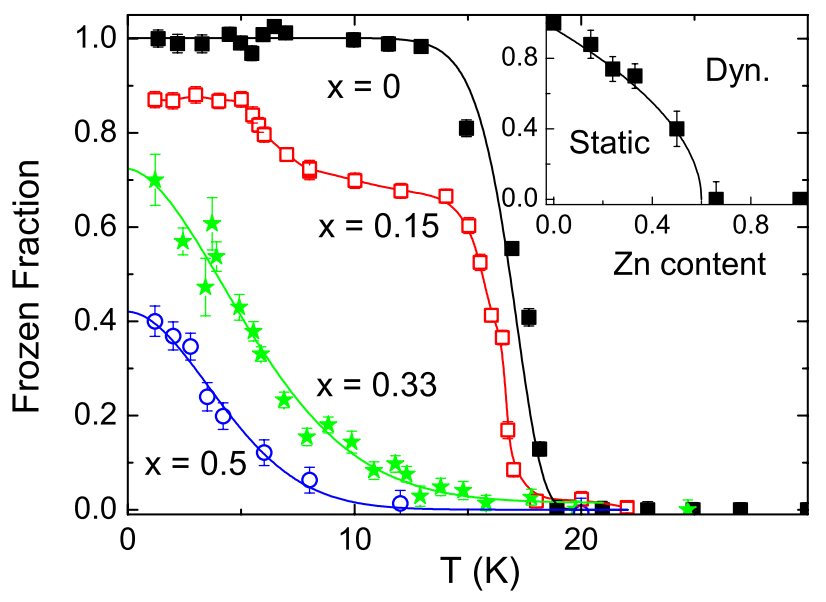

FIG. 4 (color online). $\quad T$ variation of the frozen fraction for all $x$ where a magnetic transition is observed. Inset: low- $T$ fraction versus $x$. Symbols used here are the same as in Fig. 3. Lines are guides to the eyes.

studied magnetoplumbite family [20] or of volborthite. We find no marginal spin glass state even for the 1 order of magnitude smaller $T / J$ range, and the low- $T$ relaxation is much smaller than the unconventional one observed in these spin glasslike phases $[6,8,12]$. The explanation for this cannot be related to the absence of defects since this system also displays a low- $T$ Curie tail in the SQUID data (approximately a few \%/f.u.). This statement certainly needs to be refined by a better understanding of the nature of these defects in $\mathrm{Zn}$ paratacamite.

Two scenarios could be at work to explain the low- $T$ dynamical properties. The first one traces back to the RVB initial picture or as well to any singlet ground state [5]. If the singlet-triplet gap is large as compared to the $T$ range of the data presented here, one should not detect any dynamics through the muon, which senses magnetic fields only. The remaining dynamics could be due to singlets which are broken by the defects and would yield either deconfined unpaired spins (sporadic excitations [8]) as expected in a RVB model or localized unpaired spins, depending on the nature of the ground state. It is worth noting that we did not find any drastic change in the $\mu$ SR relaxation up to $150 \mathrm{~K}$. This does not necessarily means the absence of a paramagnetic to singlet phase transition since in these phases the dynamics is either too fast for the muon or absent and the transition might be hard or even impossible to detect through $\mu \mathrm{SR}$. In this scenario, the absence of a sizeable change in the relaxation between the $x=0.66$ and 1 samples, as compared to other kagomé compounds $[8,12]$, could be well explained by an unchanged concentration of defects in the kagomé plane since only the apical $\mathrm{Zn}$ is replaced by $\mathrm{Cu}$ when $x$ is decreased. The details of the interactions between defects and apical $\mathrm{Cu}^{2+}$ ions might govern the low- $T$ dynamics and could be responsible for the small change observed in the relaxation rate. The second scenario relies on the idea that the singlet-triplet gap is smaller than $50 \mathrm{mK}$, despite a $300 \mathrm{~K} J$ value. The density of excitations would still be quite high, and $\mathrm{ZnCu}_{3}(\mathrm{OH})_{6} \mathrm{Cl}_{2}$ would then reproduce the essential features of a "cooperative paramagnet" such as observed in $\mathrm{Tb}_{2} \mathrm{Ti}_{2} \mathrm{O}_{7}$ spin liquid state [7] which has no transition down to $70 \mathrm{mK}$. However, these systems may only be loosely related since $\mathrm{Tb}_{2} \mathrm{Ti}_{2} \mathrm{O}_{7}$ with large and Ising-like spins likely belongs to another class of frustrated magnets, especially if one refers to the ordered spin-ice behavior found in the parent $\mathrm{Tb}_{2} \mathrm{Sn}_{2} \mathrm{O}_{7}$ compound [21,22].

In conclusion, $\mathrm{Zn}$ paratacamite is a very promising candidate for the $S=1 / 2$ kagomé lattice model. The issues of the existence of a gap and of the nature of low energy excitations will certainly be major avenues for future research in the field of GFM. The explanation of the different dynamical behaviors and ground states of various kagomé-like compounds will certainly represent a second challenge and not only is the $x=1.0$ compound interesting but the broad range of $x$ values above $0.5-0.6$ are also, which provide a way of probing the physics through perturbative 3D couplings.

P. M. thanks C. Lhuillier for bringing $\mathrm{Zn}$ paratacamite to his attention. This work was supported by the EC FP 6 program, Contract No. RII3-CT-2003-505925. M. A. d. V. acknowledges support from the HFM network of the ESF.

[1] P. W. Anderson, Mater. Res. Bull. 8, 153 (1973).

[2] P. Lecheminant et al., Phys. Rev. B 56, 2521 (1997).

[3] F. Mila, Phys. Rev. Lett. 81, 2356 (1998).

[4] B. Canals and C. Lacroix, Phys. Rev. Lett. 80, 2933 (1998).

[5] G. Misguich and C. Lhuillier, in Frustrated Spin Systems, edited by H. T. Diep (World Scientific, Singapore, 2005).

[6] Y. J. Uemura et al., Phys. Rev. Lett. 73, 3306 (1994).

[7] J.S. Gardner et al., Phys. Rev. Lett. 82, 1012 (1999).

[8] D. Bono et al., Phys. Rev. Lett. 93, 187201 (2004).

[9] Y. Narumi et al., Europhys. Lett. 65, 705 (2004).

[10] J. C. Domenge, P. Sindzingre, C. Lhuillier, and L. Pierre, Phys. Rev. B 72, 024433 (2005).

[11] Z. Hiroi et al., J. Phys. Soc. Jpn. 70, 3377 (2001).

[12] A. Fukaya et al., Phys. Rev. Lett. 91, 207603 (2003).

[13] F. Bert et al., Phys. Rev. Lett. 97, 117203 (2006).

[14] X. G. Zheng et al., Phys. Rev. Lett. 95, 057201 (2005).

[15] M. P. Shores, E. A. Nytko, B. M. Barlett, and D. G. Nocera, J. Am. Chem. Soc. 127, 13462 (2005).

[16] A. Schenck and K. M. Crowe, Phys. Rev. Lett. 26, 57 (1971).

[17] Following J. S. Lord, S. P. Cottrell, and W. G. Williams, Physica (Amsterdam) 289B-290B, 495 (2000), we find $\mathrm{P}_{\mathrm{OH}}(t)=P_{0}\left(\frac{1}{6}+\frac{1}{3} \cos \frac{\omega t}{2}+\frac{1}{6} \cos \omega t+\frac{1}{3} \cos \frac{3 \omega t}{2}\right)$.

[18] Rigorously, one relaxation rate per site should be introduced, but the fits do not allow a reliable determination.

[19] P. Mendels, D. Bono, and A. Wills (unpublished).

[20] A. P. Ramirez, G. P. Espinosa, and A. S. Cooper, Phys. Rev. Lett. 64, 2070 (1990).

[21] I. Mirebeau et al., Phys. Rev. Lett. 94, 246402 (2005).

[22] F. Bert et al., Phys. Rev. Lett. 97, 117203 (2006).

[23] Polarizations are deduced ( \pm 0.05 ) by subtracting a background from raw asymmetries and normalizing to 1 . 